SOCIOECONOMIC DIFFERENCES IN THE ADOPTION OF NEW MEDICAL TECHNOLOGIES

Dana Goldman

James P. Smith

Working Paper 11218 
NBER WORKING PAPER SERIES

\title{
SOCIOECONOMIC DIFFERENCES IN THE ADOPTION OF NEW MEDICAL TECHNOLOGIES
}

\author{
Dana Goldman \\ James P. Smith \\ Working Paper 11218 \\ http://www.nber.org/papers/w11218 \\ NATIONAL BUREAU OF ECONOMIC RESEARCH \\ 1050 Massachusetts Avenue \\ Cambridge, MA 02138 \\ March 2005
}

The expert programming assistance of Abby Albert and Patty St. Clair is gratefully acknowledged. This research was supported by a grant from NIA. The views expressed herein are those of the author(s) and do not necessarily reflect the views of the National Bureau of Economic Research.

(C) 2005 by Dana Goldman and James P. Smith. All rights reserved. Short sections of text, not to exceed two paragraphs, may be quoted without explicit permission provided that full credit, including $\odot$ notice, is given to the source. 
Socioeconomic Differences in the Adoption of New Medical Technologies

Dana Goldman and James P. Smith

NBER Working Paper No. 11218

March 2005

JEL No. D6, H0

\begin{abstract}
New medical technologies hold tremendous promise for improving population health, but they also raise concerns about exacerbating already large differences in health by socioeconomic status (SES). If effective treatments are more rapidly adopted by the better educated, SES health disparities may initially expand even though the health of those in all groups eventually improves. Hypertension provides a useful case study. It is an important risk factor for developing cardiovascular disease, the condition is relatively common, and there are large differences in rates of hypertension by education. This paper examines the short and long-term diffusion of two important classes of anti-hypertensives - ACE inhibitors and calcium channel blockers - over the last twenty-five years. Using three prominent medical surveys, we find no evidence that the diffusion of these drugs into medical practice favored one education group relative to another. The findings suggest that - at least for hypertension - SES differences in the adoption of new medical technologies are not an important reason for the SES health gradient.

\author{
Dana Goldman \\ RAND Graduate School \\ 1700 Main Street \\ P.O. Box 2138 \\ Santa Monica, CA 90407-2138 \\ and NBER \\ dgoldman@rand.org \\ James P. Smith \\ RAND \\ 1700 Main Street \\ P.O. Box 2138 \\ Santa Monica, CA 90407-2138 \\ james_smith@rand.org
}


New medical technologies hold tremendous promise for improving population health, but they also raise concerns about exacerbating already large differences in health by socioeconomic status (SES) (James P. Smith, 1999). In prior work, we found that better self-management of disease by the more educated was a critical factor in maintaining the SES health gradient for two distinct diseases—diabetes and HIV (Dana Goldman and James P. Smith, 2002). For these diseases— both characterized by the introduction of new effective treatment technologiesdifferences by education in patient adherence to prescribed treatment explained a significant component of the health gradient.

The impact of a new technology on health disparities depends not only on how difficult it is to adhere, but also on who actually receives the treatment. If effective technologies are more rapidly adopted by the better educated, SES health disparities may initially expand even though the health of those in the lowest SES groups eventually improves.

Hypertension provides a useful example. It is a major risk factor for cardiovascular disease and a relatively common condition-in 2002, $21 \%$ of those 18 and older had the disease, and the prevalence grows rapidly with age (over 50\% among those 65 and over). There also is a gradient with schooling - 29\% prevalence among those with less than a high school diploma compared to only $19 \%$ for those with a bachelor's degree or more. ${ }^{1}$ Before the introduction of effective drugs, the recommended treatment consisted of some combination of exercise and diet, particularly to reduce excessive weight and salt. But as a consequence of positive results from clinical trials, starting in the early 1970s, a sequence of very effective drug treatments has been introduced.

In this paper, we examine the short and long-term diffusion of anti-hypertensive therapy and how that diffusion varies by a key SES marker-education. Our investigation begins with 
the Framingham Heart Study (FHS), which has contributed much of the epidemiological knowledge about cardiovascular disease and its risk factors. Initiated in 1948, the FHS enrolled 5,209 white men and women aged 28 to 62 from Framingham, Massachusetts. Framingham is well suited to our analysis as it begins with a cohort free of cardiovascular disease in 1948 and follows them for over 50 years, tracking their health status with biennial medical examinations. Systolic and diastolic blood pressure are measured three times at each examination, and a physician performs a comprehensive medical examination. Patients are also asked to list all medications they are taking with the list of medications changing over time as new drugs enter the market. ${ }^{2}$ As the original cohort progressed, many FHS participants developed hypertension and initiated drug treatment.

Table 1 documents patterns of diffusion of anti-hypertensive therapies among diagnosed hypertensives in FHS, including diuretics, beta-blockers, calcium channel blockers, and ACE inhibitors. In 1978, less than half of FHS hypertensives were taking any drug at all and virtually all these drugs were in the diuretic class. Clinical evidence was first provided for the efficacy of diuretics in the late 1960's and early 1970's.

The data in Table 1 inform us that the use of drugs to treat hypertension has grown rapidly over these sixteen years and is no doubt a key factor contributing to the substantial decline in heart disease risk and mortality. This expansion partly reflects the increased use of existing drugs, but also the adoption of a series of new drug innovations hitting the market. Following FDA approval for hypertension in 1976, beta-blockers were introduced into widespread clinical practice; by 1978, $10 \%$ of the Framingham hypertensives were using the drug. Usage rate doubled over the next four years and plateaued around $30 \%$ by the late 1980 s. FHS first asked about calcium channel blockers in 1982, but their use received a big boost when 
they were recommended by a national panel of experts in 1988 as an option for first line therapy (JNC IV, 1988). Finally, ACE inhibitors first appear in the FHS in 1986 and were quickly adopted in the late 1980s and early 1990s. These last two drugs in particular appear to both expand overall drug use but also to substitute to some extent for older antihypertensives.

Our principal question concerns the relative speed of adoption of these new drug therapies of people with different education levels. These patterns are described in Table 2 . Is it the case that more educated patients adopt new drugs at a more rapid rate? The two most interesting drugs for our purposes are calcium channel blockers and ACE inhibitors, since we can observe the full adoption paths. For neither of them do there appear to be any systematic differences by schooling in the speed of adoption.

As a more formal test, using the FHS we estimated linear regression models for the use of each drug where the covariates included age, gender, marital status, the education classes used in Table 2, mean cholesterol levels in the exam, blood sugar level, whether the person was obese or overweight, and a quadratic time trend. This quadratic time trend was interacted with the education dummies to see if the speed of diffusion varied by schooling level. The p-values for the $\mathrm{F}$ tests for differences across education groups were 0.87 (ACE inhibitors), 0.37 (calcium channel blockers), 0.38 (beta-blockers), 0.04 (diuretics), and 0.39 (taking any drug). The only statistically significant effect was obtained for diuretics, and in that case the least educated were more likely to be taking the drug.

Framingham may be unique for several reasons. It is a closed, aging cohort that represents one relatively homogenous New England town (for example no racial minorities). Moreover, doctors rather than patients may drive the adoption process, and doctors in this midsize town may have simply recommended the same drug therapy regimen to all their patients 
regardless of their education. Even though a relationship of SES and adoption may not exist in Framingham or any other specific place, an aggregate relation could emerge if communities whose average SES levels are higher than average adopt new medical technologies more quickly (perhaps due to the type of doctors who work there).

To test this hypothesis, we turn to the National Ambulatory Medical Care Survey (NAMCS), a nationally representative study of ambulatory care in physicians' offices. Each year 3,000 physicians are randomly selected to provide data on approximately 30 patient visits over a 1-week period. Data are obtained on patients' symptoms, physicians' diagnoses, and medications ordered or provided. Since there is no information on individual patients' SES, we use area-specific mean education to detect whether adoption was faster in high SES locales. Geography is coarse in NAMCS so we are limited to the four census regions and within regions to whether it is a MSA or not. Since drug data are only available starting in 1980 and we want to track the full diffusion, we focus on calcium channel blockers and ACE inhibitors used by diagnosed hypertensives.

Figures 1.a and 1.b plot the diffusion of calcium channel blockers and ACE inhibitors for the four census regions. For both drugs, there exists a remarkable similarity in the speed of adoption across geography. To provide a more formal test, we estimated a model predicting the use of each drug by year on a quadratic time trend and region-urban dummies with interactions of that quadratic with each of the eight sub-regions (the four Census regions subdivided into its MSA and non-MSA components). The $\mathrm{p}$ values for the F-tests of no statistically significant differences in trends across these sub-regions was $\mathrm{p}=0.57$ for calcium channel blockers and $\mathrm{p}=0.11$ for ACE inhibitors. Subject to the broad level of geographic aggregation forced by 
available data, we find no evidence of any relationship between the area specific levels of education and the speed of diffusion of hypertensive drugs.

Finally, we turn our attention to a nationally representative sample of hypertensives from the third wave of the National Health and Nutrition Examination Survey (NHANES III) fielded between 1988 and 1994. NHANES III contains data obtained through personal interviews and physical exams for 33,994 people two months and older. Information is available on the prevalence of a wide variety of illnesses including hypertension, specific types of drugs taken, and individual characteristics including age, gender, race, marital status, household income and education. Besides the four Census regions and an urban rural distinction within each region, one can identify 35 separate large counties. For each of these 43 identified areas, we computed mean education levels of those 25-64 years old from the 1990 Census and matched it to the NHANES III files. Although individual years cannot be identified, the data are separated into two nationally representative phrases (1988-1991 and 1991-1994).

We estimated models predicting the use of calcium channel blockers and ACE inhibitors for people with hypertension. These models included measures of the demographic variables listed above, health co-morbidities (serum cholesterol, serum glucose, diabetes, arthritis, bronchitis, asthma, cancer, and heart disease) and health behaviors (smoking). For our purpose, the critical variables are household income and individual education (classified as not a high school graduate, high school grad, and more than high school). Household income was never statistically significant, and the p-values for the F-tests for education were 0.65 for channel betablockers and 0.45 for ACE inhibitors. The results for these SES variables did not change if we limited the sample to those hypertensives taking medication or to the first phase of NHANES III (spanning 1988 to 1991 only). These models also included mean level of education in the 
identifiable areas. In no case was area-specific, mean education levels related to the adoption of any of the drugs, providing additional evidence that a relationship between education and adoption emerges as more educated areas adopted more quickly.

\section{Conclusions}

The reasons for the very large difference in health outcomes by several measures of SES are being vigorously debated anew within and outside economics (Smith, 2005). In this paper, we investigated one possible reason for the health gradient—-that novel and effective medical technologies are adopted more quickly by those with higher SES, giving them at least a temporary advantage in improved health. We test this hypothesis by examining the introduction of new drugs to treat hypertension, of which there have been several important examples over the last twenty-five years. However, using three prominent medical surveys we find no evidence that the diffusion of these drugs into medical treatment favored one education group relative to another. This suggests that at least for hypertension, a key risk factor for heart disease, the SES differences in the adoption of new medical technologies are not an important reason for the SES health gradient.

There are several caveats to our conclusions. We have only analyzed a single medical condition, one where the short-term medical risks are not great, the recommended drug therapies not particularly expensive, and where the therapeutic efficacy of some of the new drug innovations has been questioned ex post. Whether our findings reported here extend to other diseases, especially those that are more severe and/or more expensive to treat so that people with higher incomes may have an extra advantage, must await additional research by others and us. 


\section{References}

Goldman, Dana, and Smith James P. "Can Patient Self-Management Help Explain the SES Health Gradient?" Proceedings of the National Academy of Sciences; USA (PNAS), August 6,2002 99(16): pp. 10929-10934.

Joint National Committee. "The 1988 Report of the Joint National Committee on Detection, Evaluation, and Treatment of High Blood Pressure.” Archives of Internal Medicine, 1988 148: pp.1023-1038.

Smith James P. "Healthy Bodies and Thick Wallets," Journal of Economic Perspectives, 1999 13(2) pp.145-166.

Smith, James P. "Unraveling the SES Health Connection," Population and Development Review, Spring 2005 forthcoming. 
Table 1

Fraction of Hypertensives Taking Antihypertensives by Year

\begin{tabular}{cccccc}
\hline Exam Year & Any Drug & Diuretics & Beta Blockers & Calcium & ACE inhibitors \\
1978 & 0.49 & 0.45 & 0.10 & & \\
1980 & 0.53 & 0.48 & 0.13 & & \\
1982 & 0.56 & 0.49 & 0.20 & 0.01 & \\
1984 & 0.61 & 0.50 & 0.26 & 0.03 & \\
1986 & 0.64 & 0.49 & 0.24 & 0.06 & 0.05 \\
1988 & 0.83 & 0.52 & 0.30 & 0.17 & 0.15 \\
1990 & 0.86 & 0.46 & 0.30 & 0.22 & 0.22 \\
1992 & 0.88 & 0.45 & 0.32 & 0.35 & 0.31 \\
1994 & 0.85 & 0.38 & 0.31 & 0.32 & 0.33 \\
\hline
\end{tabular}

Source- Framingham Heart Study 
Table 2

Education Gradient in Antihypertensive Drug Use

\begin{tabular}{|c|c|c|c|c|c|c|c|c|c|}
\hline \multicolumn{10}{|c|}{ Any Drug } \\
\hline Education & 1978 & 1980 & 1982 & 1984 & 1986 & 1988 & 1990 & 1992 & 1994 \\
\hline 8 or less & 0.50 & 0.55 & 0.59 & 0.65 & 0.70 & 0.87 & 0.87 & 0.93 & 0.87 \\
\hline Some HS & 0.50 & 0.54 & 0.57 & 0.59 & 0.65 & 0.84 & 0.93 & 0.87 & 0.85 \\
\hline HS degree/SC & 0.48 & 0.52 & 0.56 & 0.60 & 0.63 & 0.82 & 0.84 & 0.86 & 0.85 \\
\hline College degree & 0.46 & 0.52 & 0.54 & 0.59 & 0.60 & 0.85 & 0.87 & 0.86 & 0.87 \\
\hline \multicolumn{10}{|c|}{ Diuretics } \\
\hline Education & 1978 & 1980 & 1982 & 1984 & 1986 & 1988 & 1990 & 1992 & 1994 \\
\hline 8 or less & 0.47 & 0.51 & 0.54 & 0.56 & 0.55 & 0.58 & 0.47 & 0.51 & 0.43 \\
\hline Some HS & 0.46 & 0.49 & 0.48 & 0.44 & 0.51 & 0.50 & 0.44 & 0.47 & 0.40 \\
\hline HS degree/SC & 0.46 & 0.47 & 0.47 & 0.47 & 0.46 & 0.47 & 0.42 & 0.42 & 0.35 \\
\hline College degree & 0.42 & 0.46 & 0.45 & 0.48 & 0.45 & 0.55 & 0.52 & 0.44 & 0.39 \\
\hline
\end{tabular}

\begin{tabular}{lccccccccc}
\hline & \multicolumn{8}{c}{ Beta Blockers } \\
Education & 1978 & 1980 & 1982 & 1984 & 1986 & 1988 & 1990 & 1992 & 1994 \\
\hline 8 or less & 0.09 & 0.10 & 0.19 & 0.25 & 0.24 & 0.27 & 0.31 & 0.36 & 0.35 \\
Some HS & 0.09 & 0.10 & 0.17 & 0.22 & 0.21 & 0.28 & 0.30 & 0.31 & 0.35 \\
HS degree/SC & 0.10 & 0.15 & 0.21 & 0.28 & 0.27 & 0.32 & 0.32 & 0.31 & 0.28 \\
College degree & 0.11 & 0.13 & 0.20 & 0.28 & 0.23 & 0.30 & 0.26 & 0.29 & 0.32 \\
\hline & & & \multicolumn{7}{c}{ Calcium } \\
Education & 1978 & 1980 & 1982 & 1984 & 1986 & 1988 & 1990 & 1992 & 1994 \\
\hline 8 or less & & & 0.01 & 0.04 & 0.06 & 0.15 & 0.21 & 0.30 & 0.28 \\
Some HS & & & 0.03 & 0.05 & 0.10 & 0.22 & 0.34 & 0.40 & 0.37 \\
HS degree/SC & & & 0.01 & 0.03 & 0.07 & 0.19 & 0.22 & 0.29 & 0.36 \\
College degree & & & 0.00 & 0.02 & 0.03 & 0.10 & 0.16 & 0.30 & 0.23 \\
\hline
\end{tabular}

\begin{tabular}{|c|c|c|c|c|c|c|c|c|c|}
\hline \multirow[b]{2}{*}{ Education } & \multicolumn{8}{|c|}{ Ace Inhibitors } & \multirow[b]{2}{*}{1994} \\
\hline & 1978 & 1980 & 1982 & 1984 & 1986 & 1988 & 1990 & 1992 & \\
\hline 8 or less & & & & & 0.05 & 0.14 & 0.22 & 0.27 & 0.33 \\
\hline Some HS & & & & & 0.02 & 0.09 & 0.17 & 0.17 & 0.25 \\
\hline HS degree/SC & & & & & 0.05 & 0.17 & 0.23 & 0.28 & 0.34 \\
\hline College degree & & & & & 0.05 & 0.16 & 0.26 & 0.29 & 0.37 \\
\hline
\end{tabular}

Source- Framingham Heart Study 


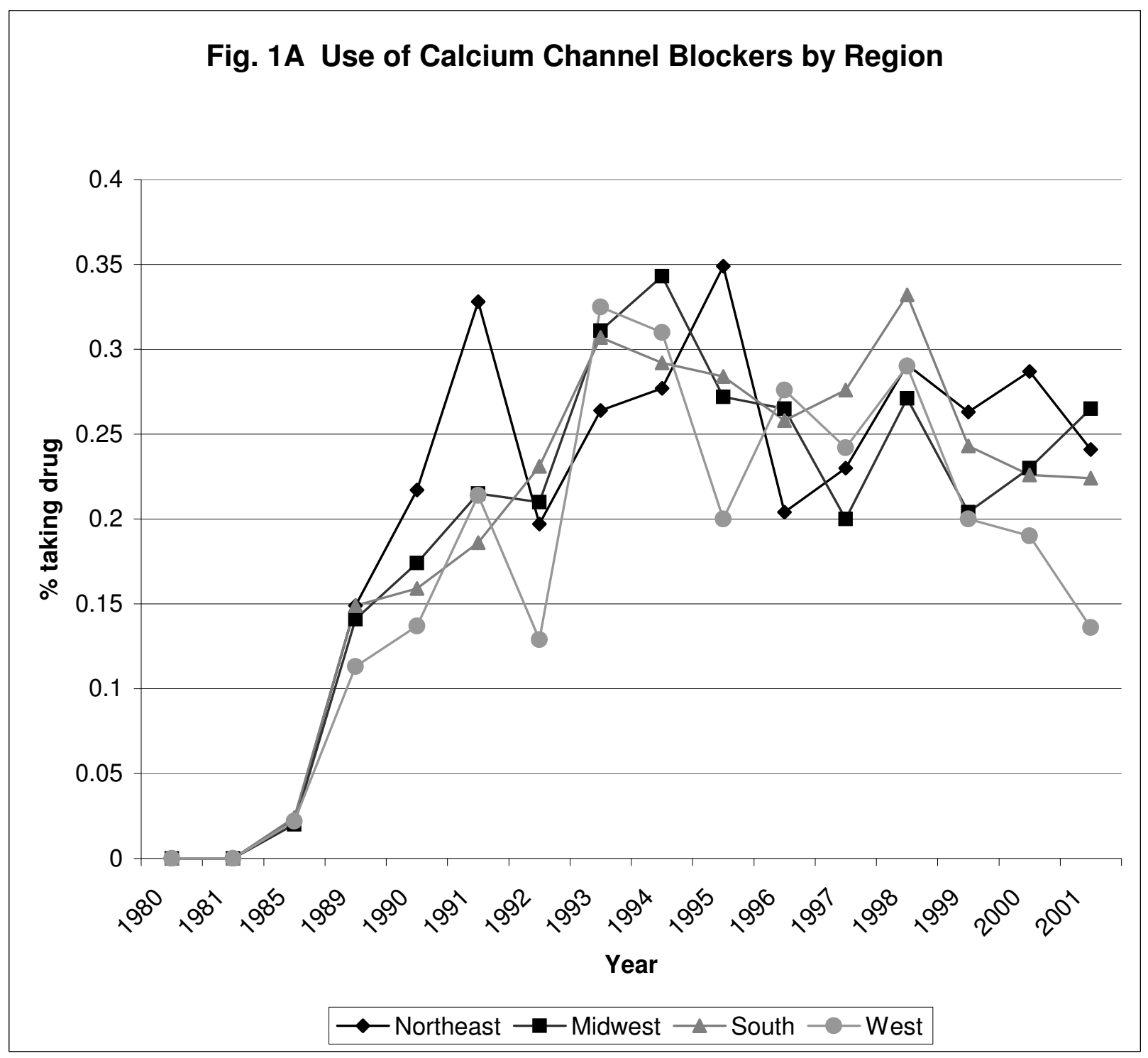




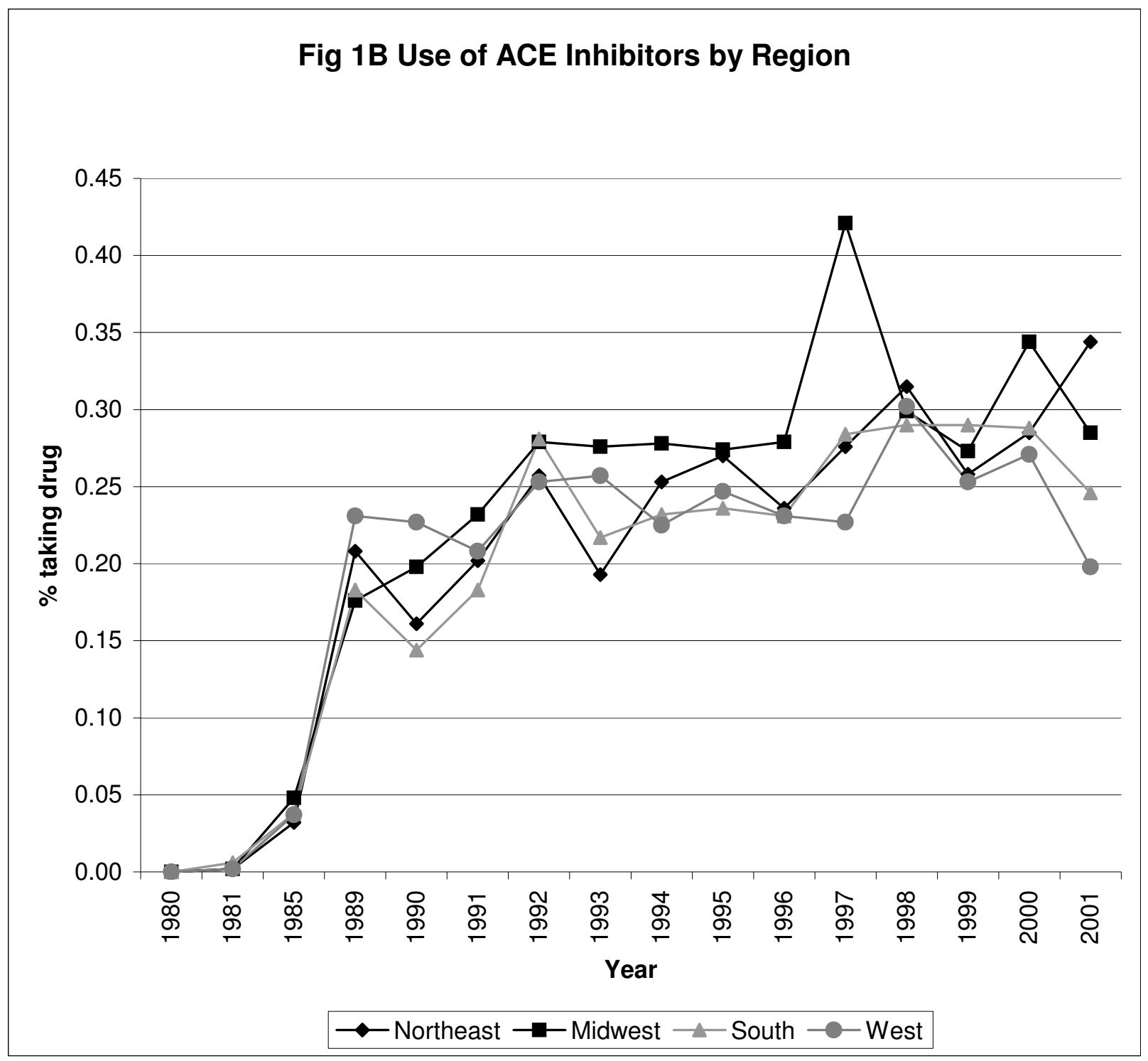




\section{Endnotes}

${ }^{1}$ All data are from National Center for Health Statistics Stat 10 (222) 2004, see Table 2.

${ }^{2}$ Prior to Exam 14 the FHS did not contain any specific class of anti-hypertensives drugs other than diuretics. Starting with Exam 14, the FHS added propranolol, the earliest beta-blocker. Eventually, FHS added calcium channel blockers and one ACE inhibitor, as well as betablockers as a class. 